\title{
RETRACTED ARTICLE: Improved cuckoo search algorithm using dimensional entropy gain
}

\author{
Qian Zhang • Lin Wang · Jun Cheng •
}

Renlong Pan

Received: 8 April 2014/ Accepted: 16 October 2014/Published online: 15 November 2014

(C) The Natural Computing Applications Forum 2015

The authors and the Editor-in-Chief have retracted this article due to the failure of the authors to secure permission for the re-use of the cuckoo search algorithm introduction.

Q. Zhang $(\varangle) \cdot$ L. Wang $\cdot$ J. Cheng $\cdot$ R. Pan

Pattern Recognition and Intelligent Systems Key Laboratory of Guizhou, Guiyang 550025, Guizhou, China

e-mail: gzmuzq@gmail.com

Q. Zhang $\cdot$ L. Wang

School of Computer Science and Engineering of Guizhou Minzu

University, Guiyang 550025, Guizhou, China 